Smart Infrastructure and Construction Volume 170 Issue SC2

Patch antenna sensor rosettes for surface strain measurement

Li, Cho and Wang
Proceedings of the Institution of Civil Engineers

Smart Infrastructure and Construction 170 June 2017 Issue SC2

Paper 1700004

Received 22/01/2017 Accepted 21/06/2017

Published online 18/07/2017

Keywords: health \& safety/modelling/strain
Pages 39-49 http://dx.doi.org/10.1680/jsmic.17.00004

\title{
Patch antenna sensor rosettes for surface strain measurement
}

1 Dan Li BS, MS

PhD student, School of Civil and Environmental Engineering, Georgia Institute of Technology, Atlanta, GA, USA

2 Chunhee Cho PhD

Post-doctoral Researcher, Department of Civil and Environmental Engineering and Construction, University of Nevada, Las Vegas, NV, USA; School of Civil and Environmental Engineering, Georgia Institute of Technology, Atlanta, GA, USA
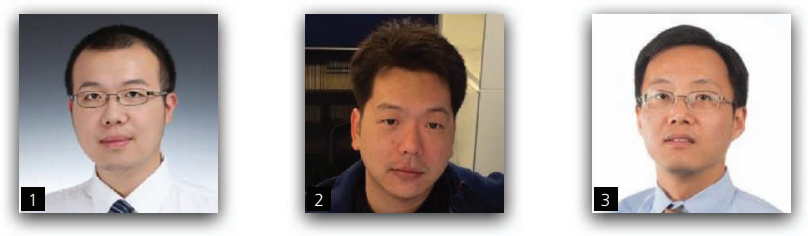

$3 \quad$ Yang Wang PhD

Associate Professor, School of Civil and Environmental Engineering, Georgia Institute of Technology, Atlanta, GA, USA (corresponding author: yang.wang@ce.gatech.edu)

Passive (battery-free) wireless patch antenna sensors have been developed in recent years for strain sensing, to provide convenient and low-cost instrumentation. Despite past efforts, current analytical and experimental studies have mainly focused on performance in single-axial measurement, which is simple and unrealistic from typically encountered arbitrary plane stress fields. This paper presents strain sensor rosettes made of folded patch antennas and slotted patch antennas for measuring an arbitrary surface strain (plane stress) field. The transverse strain effect of both sensors is discussed and has been validated through laboratory experiments. Multiphysics coupled simulation is conducted to describe accurately the mechanical and electromagnetic behaviours of antenna sensors. Resonance frequency shifts of the antenna sensors are used to derive the three strain components in an arbitrary plane stress scenario - that is, two normal and one shear strain components. Both numerical studies and experimental validations have been performed.

\section{Notation}

c speed of light

$f_{0} \quad$ resonance frequency of a patch antenna sensor without strain

$f_{\epsilon} \quad$ resonance frequency of a patch antenna sensor under strain

$L^{\prime} \quad$ additional length due to fringing effect

$L_{1}, L_{\mathrm{t}} \quad$ lengths of current path of a patch antenna sensor in its longitudinal direction and transverse directions, respectively

$L_{\text {total }} \quad$ total length of current path of a patch antenna sensor

S strain sensitivity matrix of a strain sensor rosette $S_{\mathrm{l}}, S_{\mathrm{t}} \quad$ strain sensitivities of a patch antenna sensor in its longitudinal and transverse directions, respectively $\mathrm{T}$ transformation matrix of a strain sensor rosette

$\beta_{\mathrm{r}, \text { eff }} \quad$ effective dielectric constant of the substrate

$\Delta f \quad$ change in resonance frequency of a patch antenna sensor

$\Delta f \quad$ resonance frequency change vector

$\epsilon \quad$ strain vector

$\widetilde{\epsilon}_{1}, \widetilde{\epsilon}_{\mathrm{t}} \quad$ strains of a patch antenna sensor in its longitudinal direction and transverse directions, respectively $\epsilon_{1}, \epsilon_{\mathrm{t}} \quad$ strains of the base structure under a patch antenna sensor in the sensor's longitudinal and transverse directions, respectively

$\epsilon_{x}, \epsilon_{y}, \epsilon_{x y}$ strain components of the base structure under Cartesian coordinates

$\eta_{\mathrm{l}}, \eta_{\mathrm{t}} \quad$ strain transfer ratios of a patch antenna sensor in its longitudinal and transverse directions, respectively

$\theta_{i} \quad$ angle between the $x$ axis and the longitudinal direction of sensor $i$ in a strain sensor rosette $(i=1$, 2, 3)

\section{Introduction}

Strain is one of the most important physical indicators that can quantify the performance and condition of civil structures. Traditional strain-sensing systems usually utilise cable-connected equipment for data acquisition, such as metal foil strain gages and fibre-optic sensors. As a result, these systems suffer from high installation cost and inconvenient application when many sensors need to be installed over a large-scale structure (Spencer et al., 2004). The emergence of wireless sensors, in particular recently developed passive wireless sensors which do not need battery or other onboard power supply, has shown promise to overcome the cable-related difficulties (Butler et al., 2002; Tan et al., 2008). 
One example of passive wireless strain sensors is the microstrip patch antenna sensor, whose resonance frequency changes when strain is applied. Tata et al. (2009) investigated the potential of a rectangular microstrip patch antenna on strain sensing. First, by using cabled measurement, the linear relationship between resonance frequency of the antenna and strain is validated (Tata et al., 2009). The relationship is later verified by wireless interrogation (Deshmukh and Huang, 2010). As the rectangular microstrip patch antenna is sensitive to strain only along the longitudinal direction or the transverse direction, Daliri et al. (2012) proposed a circular microstrip patch antenna sensor that responds to surface strain. However, the sensor cannot distinguish the three surface strain components when an arbitrary strain field is applied.

Another widely investigated approach for passive wireless sensing is radio-frequency identification (RFID) technology. RFID technology can offer the ability to modulate the response signal from the sensor (EPCglobal Inc., 2008) and thus distinguish it from environmental reflection. In recent years, many passive wireless strain sensors based on RFID technology have been proposed. A printed RFID patch antenna has been shown to measure high strain with change in antenna gain and impedance (Merilampi et al., 2011). Meanwhile, Occhiuzzi et al. (2011) demonstrated the relationship between strain and electromagnetic behaviours of a meander-line RFID antenna sensor. In order to reduce the size of an RFID patch antenna sensor, Yi et al. (2011) proposed an antenna folding technique using vias and validated the performance of the folded patch antenna sensor by tensile experiments. To achieve further size reduction of the RFID patch antenna sensor, slots are added on the top copper cladding to provide a detoured current path, the length of which determines antenna resonance frequency (Yi et al., 2013a, 2013b). Multiphysics simulation coupling mechanics and electromagnetics is proposed to describe more accurately the behaviour of the sensor (Yi et al., 2013a, 2013b).

Although the achievements in previous research have shown great potential in wireless strain sensing, most of the studies address only scenarios when either longitudinal or transverse strain is applied separately. Wireless strain sensing of an arbitrary surface strain field, with three strain components applied simultaneously, has not been thoroughly investigated. In this paper, strain sensor rosettes made of folded patch antenna sensors or slotted patch antenna sensors are proposed for measuring an arbitrary surface strain field. The strain-sensing mechanism of patch antenna sensors is studied first. In order to achieve accurate strain measurements, the transverse strain effect of patch antenna sensors is investigated through laboratory experiments and a method for considering this effect is proposed. To investigate thoroughly the strain-sensing performance of both the rosettes, multiphysics simulation coupling mechanics and electromagnetics is conducted to study the electromagnetic behaviours of antenna sensors under strain. To understand the strain-sensing performance better by using patch antenna sensor rosettes, the consistency between one sensor scenario and a rosette scenario, as well as the effect of base structure dimensions, has been studied.

The rest of this paper is organised as follows. Section 2 introduces the rosette strain-sensing mechanism of patch antenna sensors. Section 3 proposes the method for considering the transverse strain effect in the rosette set-up. Section 4 presents the mechanics-electromagnetics coupled finite-element models and the strain-sensing simulation results. Finally, the paper is summarised with conclusion and future work.

\section{Rosette strain-sensing mechanism of wireless patch antenna sensors}

This section provides the quantitative relationship between the resonance frequency of a patch antenna sensor and the strain experienced by the sensor. Section 2.1 introduces the strainsensing mechanism of a single patch antenna sensor. The generic formulation is applicable to both folded patch antenna sensors and slotted patch antenna sensors. Section 2.2 presents the experimental validation of the strain-sensing mechanism of both types of sensors. Section 2.3 shows the strain rosette of two types of antenna sensors.

\subsection{Strain-sensing mechanism of a single patch antenna sensor}

Figure 1(a) illustrates the design of a folded patch antenna sensor; Figure 1(b) illustrates the design of a slotted patch antenna sensor. Each figure shows the top side of the patch antenna sensors, where the top copper cladding, matching line and RFID chip are mounted on a substrate. The bottom side of the sensor is the electronic ground plane and is also made of copper cladding. Vias through the substrate connect the top copper cladding and the electric ground in order to reduce the size of the antenna sensor. The matching line in Figure 1(a) is designed to achieve the best impedance matching between the folded patch antenna sensor and the RFID chip. The geometrical pattern of the slotted patch antenna sensor is designed to have the best impedance matching between the antenna and the RFID chip, so there is no independent matching line in the slotted patch antenna sensor. In each figure, dashed arrows illustrate the electrical current path on the top copper cladding of the antenna sensor. For the folded patch antenna sensor, the current path is a straight line; for the slotted patch antenna sensor, the current path is detoured around the slots.

The underlying physics of a patch antenna sensor for strain sensing is that the electromagnetic resonance frequency of the patch antenna changes under strain. When strain is applied on the sensor, the length of electrical current path changes accordingly, and thus the resonance frequency changes. This change in resonance frequency of the patch antenna sensor can be wirelessly detected through RFID technology (Yi et al., 2011). An RFID reader emits a wireless interrogation signal to the sensor, which in turn harnesses energy from the signal for its own operation. When the interrogation frequency equals the resonance frequency of the 


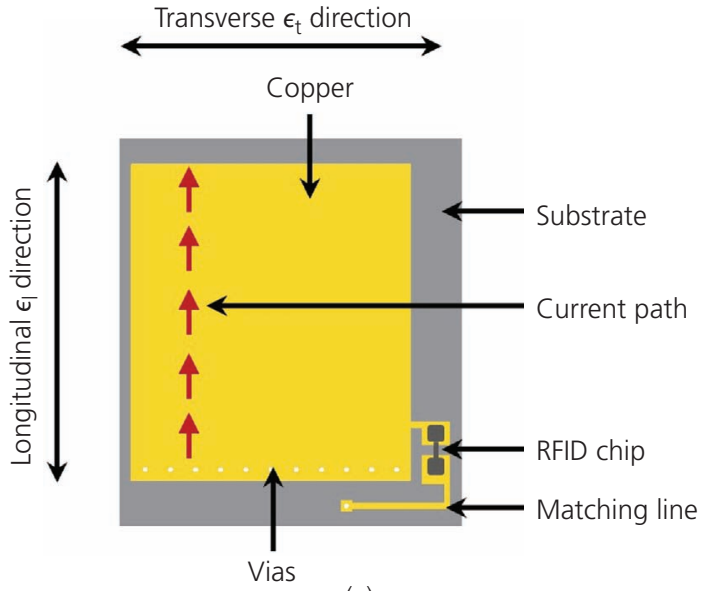

(a)

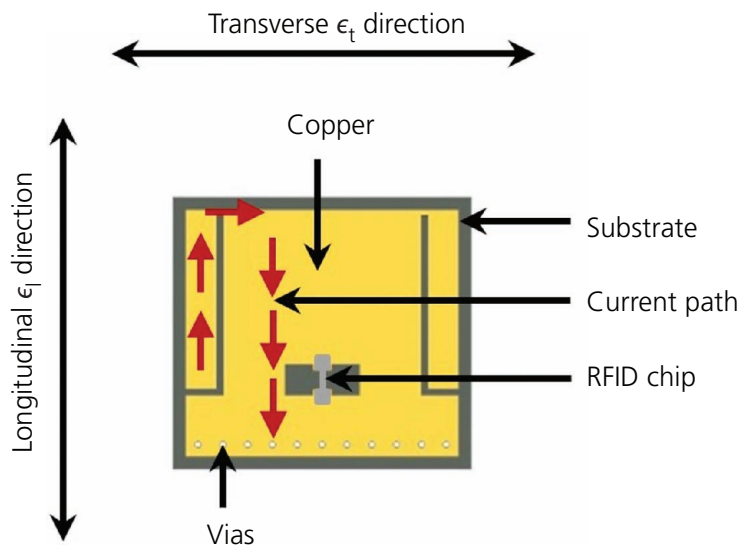

(b)

Figure 1. Illustrations of patch antenna sensors: (a) folded patch antenna $\left(69 \times 61 \times 0.787 \mathrm{~mm}^{3}\right)$; (b) slotted patch antenna $(44 \times 48 \times$ $\left.0.787 \mathrm{~mm}^{3}\right)$

patch antenna sensor, best matching between the antenna and the RFID chip occurs, and thus the least interrogation power is needed to activate the RFID chip. By recording the interrogation frequency corresponding to the minimum value of the interrogation power, the resonance frequency of the patch antenna is obtained and then used to derive the amount of strain. In general, Equation 1 shows the relationship between the resonance frequency and the geometric property of a patch antenna that has the row of vias connecting top copper cladding to the ground plane

1.

$$
f_{0}=\frac{c}{2\left(L_{1}+L_{\mathrm{t}}+L^{\prime}\right) \sqrt{\beta_{\mathrm{r}, \mathrm{eff}}}}=\frac{c}{2 L_{\mathrm{total}} \sqrt{\beta_{\mathrm{r}, \mathrm{eff}}}}
$$

where $c$ is the speed of light; $L_{1}$ is the length of current path in the longitudinal direction; $L_{\mathrm{t}}$ is the length of current path in the transverse direction; $L^{\prime}$ is the additional length due to the fringing effect; $L_{\text {total }}$ is the total length of current path; and $\beta_{\mathrm{r}, \text { eff }}$ is the effective dielectric constant. Figure 1 illustrates the longitudinal and transverse directions of each sensor. As shown in Figure 1, a folded patch antenna sensor does not have a transverse current path but a slotted patch antenna sensor has a transverse current path. For simplicity, past studies of slotted patch antenna strain sensors usually consider $L_{\mathrm{t}}$ to be much smaller than $L_{1}$ and thus do not include the current path in transverse direction (Yi et al., 2013b, 2014). However, in order to obtain an accurate strain measurement, this study will demonstrate that the transverse strain effect should be considered, particularly for slotted antenna sensors.

When the patch antenna is under relatively small longitudinal strain $\widetilde{\epsilon}_{1}$ and transverse strain $\widetilde{\epsilon}_{\mathrm{t}}$, the resonance frequency of the antenna changes to

$$
\begin{aligned}
f_{\epsilon} & =\frac{c}{2\left[L_{1}\left(1+\widetilde{\epsilon}_{1}\right)+L_{\mathrm{t}}\left(1+\widetilde{\epsilon}_{t}\right)+L^{\prime}\right] \sqrt{\beta_{\mathrm{r}, \mathrm{eff}}}} \\
& =\frac{f_{0}}{1+\left(L_{1} / L_{\text {total }}\right) \widetilde{\epsilon}_{1}+\left(L_{\mathrm{t}} / L_{\text {total }}\right) \widetilde{\epsilon}_{\mathrm{t}}} \\
& \approx f_{0}\left(1-\frac{L_{1}}{L_{\text {total }}} \widetilde{\epsilon}_{1}-\frac{L_{\mathrm{t}}}{L_{\text {total }}} \widetilde{\epsilon}_{\mathrm{t}}\right)
\end{aligned}
$$

The equation shows that the resonance frequency is approximately linear to the strain on the antenna sensor, in particular if the strain is small. Due to the bonding effect between the antenna sensor and the base structure, only some percentage of the strain in the base structure can be transferred to the antenna sensor. Longitudinal strain transfer ratio $\eta_{1}$ refers to the percentage of the longitudinal strain on the sensor $\widetilde{\epsilon}_{1}$ over the longitudinal strain on the base structure $\epsilon_{1}$; transverse strain transfer ratio $\eta_{\mathrm{t}}$ refers to the percentage of the transverse strain on the sensor $\widetilde{\epsilon}_{\mathrm{t}}$ over the transverse strain on the base structure $\epsilon_{\mathrm{t}}$. Both ratios are close to but less than $100 \%$. $\Delta f$ is used to denote the change in the resonance frequency of the antenna sensor when strain $\epsilon_{1}$ and $\epsilon_{\mathrm{t}}$ occur on the surface of the base structure. Based on the linear relationship shown in Equation 2, the relationship between $\Delta f$ and $\epsilon_{\mathrm{l}}, \epsilon_{\mathrm{t}}$ is obtained as

$$
\begin{aligned}
\Delta f & =f_{\epsilon}-f_{0} \approx-\frac{f_{0} L_{1}}{L_{\text {total }}} \widetilde{\epsilon}_{1}-\frac{f_{0} L_{\mathrm{t}}}{L_{\text {total }}} \widetilde{\epsilon}_{\mathrm{t}} \\
& =-\frac{f_{0} L_{1}}{L_{\text {total }}} \eta_{1} \epsilon_{1}-\frac{f_{0} L_{\mathrm{t}}}{L_{\text {total }}} \eta_{\mathrm{t}} \epsilon_{\mathrm{t}}=S_{1} \epsilon_{1}+S_{\mathrm{t}} \epsilon_{\mathrm{t}}
\end{aligned}
$$

where $S_{1}$ is named the longitudinal strain sensitivity and $S_{\mathrm{t}}$ is named the transverse strain sensitivity. Each sensitivity number represents the linear proportional relationship between resonance frequency and the strain along that direction. 
Smart Infrastructure and Construction

Volume 170 Issue SC2
Patch antenna sensor rosettes for surface

strain measurement

Li, Cho and Wang

\subsection{Experimental validation}

To illustrate better the transverse strain sensitivity of a patch antenna sensor, tensile tests on folded patch antenna sensors and slotted patch antenna sensors have been conducted. Figure 2(a) shows the central region of an aluminium specimen with two folded patch antenna sensors installed in perpendicular orientations. Sensor 1 has its longitudinal direction oriented along the $y$ axis (loading direction), while sensor 2 has its longitudinal direction along the $x$ axis. Likewise, Figure 2(b) shows the central region of an aluminium specimen with two slotted patch antenna sensors installed. Similarly, sensor 1 has its longitudinal direction oriented along the $y$ axis (loading direction), while sensor 2 has its longitudinal direction along the $x$ axis. Each specimen is $610 \mathrm{~mm}$ long, $150 \mathrm{~mm}$ wide and $3.2 \mathrm{~mm}$ thick. For each specimen, the loading direction is along the $y$ axis; the specimen deforms freely along the lateral $x$ direction. Ten reference metal foil strain gages are installed on each specimen, five measuring $\epsilon_{y}$ and five measuring $\epsilon_{x}$
As shown in Equation 3, strain sensitivities are mainly determined by the lengths of electric current paths. Because the actual current paths on a fabricated sensor are determined by the sensor geometry, it is preferable that the sensor fabrication is as accurate as possible. Another important factor on strain sensitivity is the strain transfer ratio. To achieve a high strain transfer ratio, strong bonding is needed when installing the antenna sensors. Other factors, such as interrogation distance, specimen size and loading method, are found to have smaller effect on the strain sensitivity of antenna sensors.

The axial force applied by the tensile machine is configured so that approximately a $+50 \mu \varepsilon$ along the $y$ axis is achieved at each loading step. Meanwhile, the specimen shrinks along the $x$ axis due to Poisson's effect. The test starts with $\epsilon_{y}=0 \mu \varepsilon$ and ends around $\epsilon_{y}=$ $350 \mu \varepsilon$. At each loading step, the resonance frequencies for both sensors and the strain for both directions are recorded.

For each specimen, Figure 3 shows the strain $\epsilon_{y}$ against $\epsilon_{x}$ measured by metal foil strain gages. The slope of each plot is

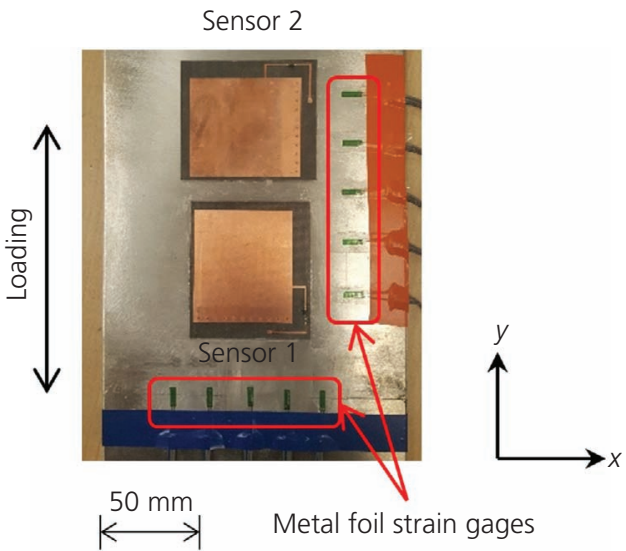

(a)

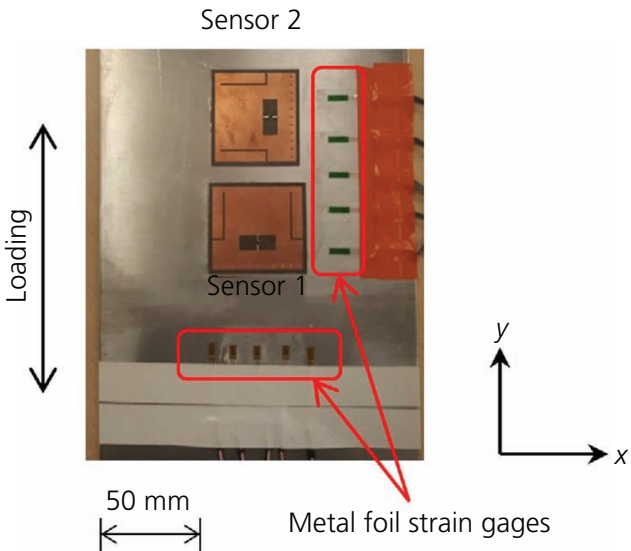

(b)

Figure 2. Sensor instrumentation for tensile test: (a) folded patch antennas; (b) slotted patch antennas

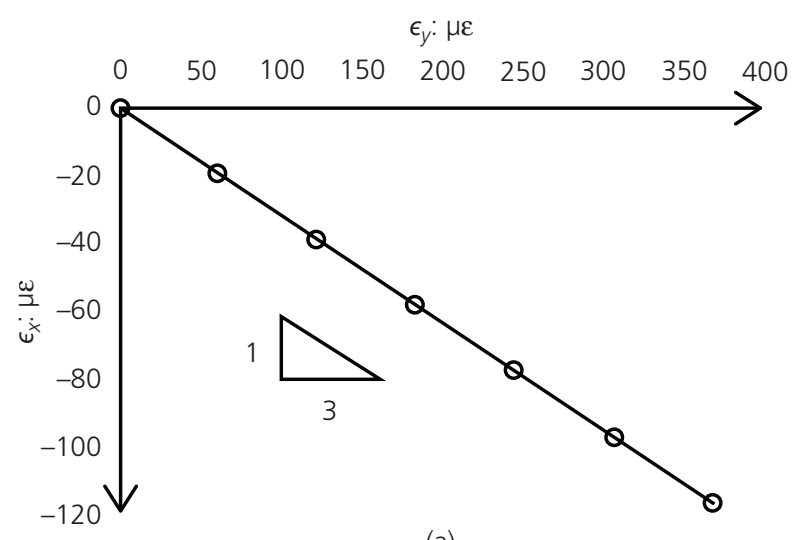

(a)

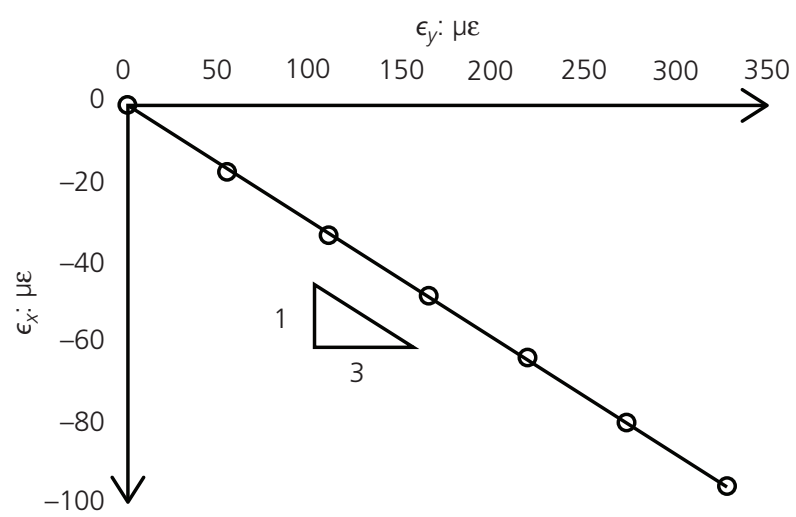

(b)

Figure 3. Strain $\epsilon_{y}$ against $\epsilon_{x}$ measured by metal foil strain gages: (a) seven loading steps for specimen with folded patch antenna sensors; (b) seven loading steps for specimen with slotted patch antenna sensors 
Smart Infrastructure and Construction

Volume 170 Issue SC2
Patch antenna sensor rosettes for surface

strain measurement

Li, Cho and Wang
Table 1. Resonance frequencies of patch antenna sensors: $\mathrm{MHz}$

\begin{tabular}{|c|c|c|c|c|}
\hline \multirow[t]{2}{*}{ Loading step } & \multicolumn{2}{|c|}{$\begin{array}{l}\text { Folded patch antenna } \\
\text { sensor }\end{array}$} & \multicolumn{2}{|c|}{$\begin{array}{l}\text { Slotted patch antenna } \\
\text { sensor }\end{array}$} \\
\hline & Sensor 1 & Sensor 2 & Sensor 1 & Sensor 2 \\
\hline 1 & 909.663 & $904 \cdot 027$ & $912 \cdot 445$ & $912 \cdot 836$ \\
\hline 2 & 909.618 & $904 \cdot 041$ & $912 \cdot 422$ & $912 \cdot 832$ \\
\hline 3 & 909.570 & 904.051 & $912 \cdot 366$ & $912 \cdot 825$ \\
\hline 4 & 909.533 & 904.076 & $912 \cdot 320$ & $912 \cdot 817$ \\
\hline 5 & 909.494 & $904 \cdot 084$ & $912 \cdot 271$ & $912 \cdot 821$ \\
\hline 6 & 909.460 & 904.091 & $912 \cdot 234$ & $912 \cdot 815$ \\
\hline 7 & $909 \cdot 382$ & $904 \cdot 114$ & $912 \cdot 199$ & $912 \cdot 795$ \\
\hline
\end{tabular}

around $1 / 3$, which is close to the Poisson's ratio of aluminium. Table 1 lists the resonance frequencies of patch antenna sensors at all loading steps. By using the strain and resonance frequency data, the longitudinal and transverse strain sensitivities of each sensor can be calculated through linear regression according to Equation 3. This leads to a least-squares problem with longitudinal and transverse strain sensitivities $S_{1}$ and $S_{\mathrm{t}}$, respectively, as optimisation variables

$$
\text { 4. } \underset{S_{1}, S_{\mathrm{t}}}{\operatorname{minimise}} \sum_{i}\left[\Delta f_{i}-\left(S_{1} \epsilon_{1 i}+S_{\mathrm{t}} \epsilon_{\mathrm{t} i}\right)\right]^{2}
$$

Upon solving the least-squares problem by using available data points, Table 2 lists the longitudinal and transverse strain sensitivities of both sensors. The folded patch antenna sensor has a transverse strain sensitivity of $25 \mathrm{~Hz} / \mu \varepsilon$, which is close to $0 \mathrm{~Hz} / \mu \varepsilon$. This is because transverse strain cannot significantly change the total length of current path for a folded patch antenna sensor. On the other hand, due to the complicated geometry of a slotted patch antenna sensor, transverse strain changes the total length of the current path of the

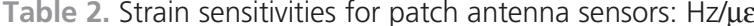

\begin{tabular}{|c|c|c|c|}
\hline Folded patch an & tenna sensor & Slotted patch a & Itenna sensor \\
\hline Longitudinal $S_{I}$ & Transverse $S_{t}$ & Longitudinal $S_{I}$ & Transverse $S_{t}$ \\
\hline$-801 \cdot 2$ & $25 \cdot 3$ & $-794 \cdot 6$ & $-362 \cdot 3$ \\
\hline
\end{tabular}

sensor. Thus, the slotted patch antenna sensor has a relatively high transverse strain sensitivity, which is around $-362 \cdot 3 \mathrm{~Hz} / \mu \varepsilon$.

\subsection{Strain rosettes of antenna sensors}

Most studies of patch antenna strain sensors have been limited to uniaxial measurement (Yi et al., 2011, 2014). In order to measure an arbitrary surface strain field, strain rosettes (Boresi and Schmidt, 2002) made of folded patch antennas or slotted patch antennas need to be deployed. Figures 4(a) and 4(b) show the rosette schematic diagram for each sensor type. Each rosette consists of three patch antenna sensors, with the three sensors rotated at $120^{\circ}$ intervals. In each rosette, the longitudinal axis of sensor 1 is placed along the $y$ axis, sensor 2 is rotated $120^{\circ}$ counterclockwise from the $y$ axis and sensor 3 is rotated $120^{\circ}$ clockwise from the $y$ axis. Because off-the-shelf RFID modulation technology mostly operates at around $900 \mathrm{MHz}$, the dimension of the antenna sensors and, thus, of the rosette is larger than a typical metal foil strain rosette. It is preferable to have a fairly uniform strain field under the rosette area, or the measurement result will be the average strain.

Considering each rosette is mounted on a flat surface area for strain measurement, the three strain components under Cartesian coordinates are denoted as $\epsilon_{x}, \epsilon_{y}$ and $\epsilon_{x y}$. They are related to the longitudinal strains of the base structure under the three sensors, $\epsilon_{1,1}, \epsilon_{1,2}$ and $\epsilon_{1,3}$, by the strain transformation equations

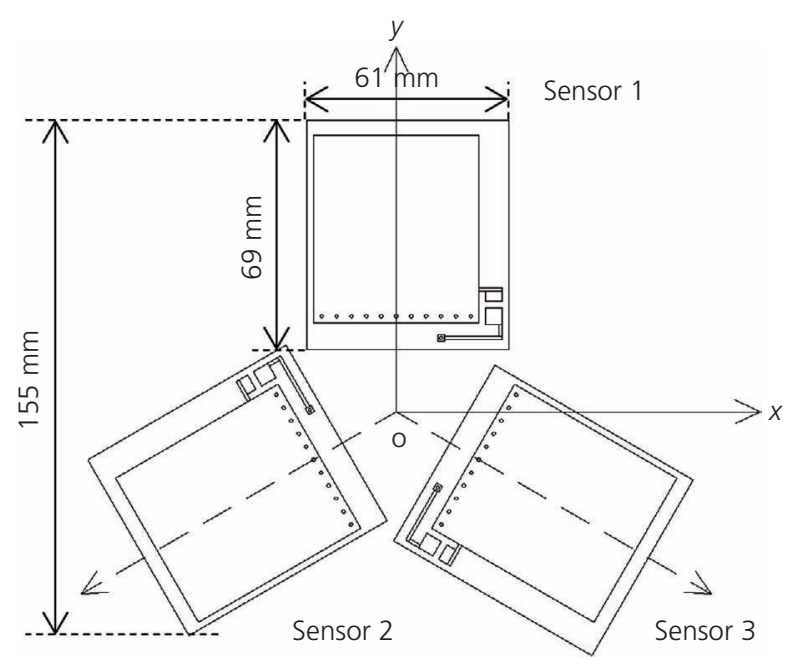

(a)

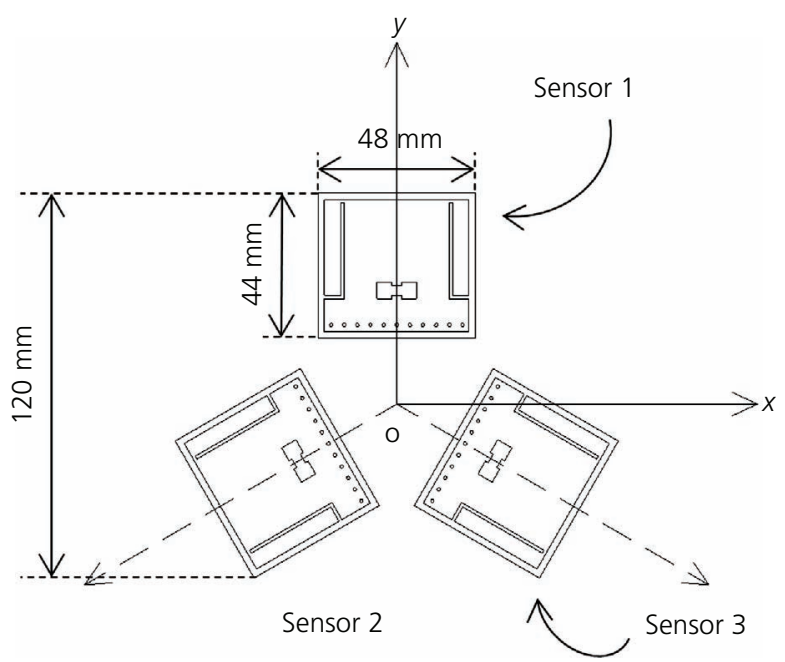

(b)

Figure 4. Schematic diagrams of the two types of strain rosettes: (a) rosette with three folded patch antennas; (b) rosette with three slotted patch antennas 


$$
\left\{\begin{array}{l}
\epsilon_{1,1} \\
\epsilon_{1,2} \\
\epsilon_{1,3}
\end{array}\right\}=\left[\begin{array}{ll}
\frac{1+\cos \left(2 \theta_{1}\right)}{2} & \frac{1-\cos \left(2 \theta_{1}\right)}{2} \sin \left(2 \theta_{1}\right) \\
\frac{1+\cos \left(2 \theta_{2}\right)}{2} & \frac{1-\cos \left(2 \theta_{2}\right)}{2} \sin \left(2 \theta_{2}\right) \\
\frac{1+\cos \left(2 \theta_{3}\right)}{2} & \frac{1-\cos \left(2 \theta_{3}\right)}{2} \sin \left(2 \theta_{3}\right)
\end{array}\right]\left\{\begin{array}{c}
\epsilon_{x} \\
\epsilon_{y} \\
\epsilon_{x y}
\end{array}\right\}
$$

5.

where $\theta_{1}$ is the angle between the $x$ axis and the longitudinal direction of sensor 1 , which equals $90^{\circ} ; \theta_{2}$ is the angle between the $x$ axis and the longitudinal direction of sensor 2, which equals $210^{\circ}$; and $\theta_{3}$ is the angle between the $x$ axis and the longitudinal direction of sensor 3 , which equals $330^{\circ}$. Therefore, in each rosette, from the longitudinal strains measured by the three sensors, the three strain components can be calculated as

6. $\left\{\begin{array}{c}\epsilon_{x} \\ \epsilon_{y} \\ \epsilon_{x y}\end{array}\right\}=\frac{1}{3}\left[\begin{array}{ccc}-1 & 2 & 2 \\ 3 & 0 & 0 \\ 0 & \sqrt{3} & -\sqrt{3}\end{array}\right]\left\{\begin{array}{c}\epsilon_{1,1} \\ \epsilon_{1,2} \\ \epsilon_{1,3}\end{array}\right\}=\mathbf{T}\left\{\begin{array}{c}\epsilon_{1,1} \\ \epsilon_{1,2} \\ \epsilon_{1,3}\end{array}\right\}$

where $\mathbf{T}$ is the transformation matrix which maps the longitudinal strains measured by three sensors onto the three strain components under Cartesian coordinates.

\section{Consideration of transverse strain effect}

When measuring a surface strain field by the strain sensor rosette, the transverse strain sensitivity of each antenna sensor should be considered. The transverse strain sensitivity in a patch antenna sensor refers to the change in resonance frequency due to strain in the transverse direction of the sensor. The transverse strain effect should be considered in order to obtain the accurate longitudinal strain value.

For an antenna sensor exposed to an arbitrary surface strain field, the change in the resonance frequency may contain contribution from both longitudinal and transverse strains. Equation 3 is repeated for convenience

7. $\Delta f=S_{1} \epsilon_{1}+S_{\mathrm{t}} \epsilon_{\mathrm{t}}$

Here $\epsilon_{1}$ is the normal strain along the longitudinal direction under the antenna sensor, $\epsilon_{\mathrm{t}}$ is the normal strain along the transverse direction under the antenna sensor, $S_{1}$ is the longitudinal strain sensitivity and $S_{\mathrm{t}}$ is the transverse strain sensitivity. It is should be noted that $S_{\mathrm{l}}$ and $S_{\mathrm{t}}$ are defined in a uniaxial strain setting rather than a uniaxial stress setting. In accordance with practical application, it is assumed that three antenna sensors in the strain rosette share the same longitudinal strain sensitivity $S_{1}$ and transverse strain sensitivity $S_{\mathrm{t}}$. By using Equations 6 and 7, the change in the resonance frequency of sensor 1 in the rosette set-up can be expressed as

$$
\begin{aligned}
\Delta f_{1} & =S_{1} \epsilon_{y}+S_{\mathrm{t}} \epsilon_{x} \\
& =S_{1} \epsilon_{1,1}+S_{\mathrm{t}}\left(\frac{2}{3} \epsilon_{1,2}+\frac{2}{3} \epsilon_{1,3}-\frac{1}{3} \epsilon_{1,1}\right) \\
& =\left(S_{1}-\frac{1}{3} S_{\mathrm{t}}\right) \epsilon_{1,1}+\frac{2}{3} S_{\mathrm{t}} \epsilon_{1,2}+\frac{2}{3} S_{\mathrm{t}} \epsilon_{1,3}
\end{aligned}
$$

Likewise, the equations for $\Delta f_{2}$ and $\Delta f_{3}$ can be derived. The change in the resonance frequencies in all three antenna sensors can be expressed as

9. $\left\{\begin{array}{c}\Delta f_{1} \\ \Delta f_{2} \\ \Delta f_{3}\end{array}\right\}=\frac{1}{3}\left[\begin{array}{ccc}3 S_{1}-S_{\mathrm{t}} & 2 S_{\mathrm{t}} & 2 S_{\mathrm{t}} \\ 2 S_{\mathrm{t}} & 3 S_{1}-S_{\mathrm{t}} & 2 S_{\mathrm{t}} \\ 2 S_{\mathrm{t}} & 2 S_{\mathrm{t}} & 3 S_{1}-S_{\mathrm{t}}\end{array}\right]\left\{\begin{array}{c}\epsilon_{1,1} \\ \epsilon_{1,2} \\ \epsilon_{1,3}\end{array}\right\}$

where $\epsilon_{1, i}$ is the longitudinal strain of the $i$ th sensor. According to Equations 6 and 9, the strain components under Cartesian coordinates can be calculated from the three frequency changes as

10. $\epsilon=\mathbf{T S}^{-1} \Delta f$

where $\boldsymbol{\epsilon}=\left[\begin{array}{lll}\epsilon_{x} & \epsilon_{y} & \epsilon_{x y}\end{array}\right]^{\mathrm{T}}$ is the strain vector, $\Delta \boldsymbol{f}=\left[\begin{array}{lll}\Delta f_{1} & \Delta f_{2} & \Delta f_{3}\end{array}\right]^{\mathrm{T}}$ is the resonance frequency change vector and $\mathbf{S}$ is the strain sensitivity matrix for Equation 9

11. $\quad \mathbf{S}=\frac{1}{3}\left[\begin{array}{ccc}3 S_{1}-S_{\mathrm{t}} & 2 S_{\mathrm{t}} & 2 S_{\mathrm{t}} \\ 2 S_{\mathrm{t}} & 3 S_{1}-S_{\mathrm{t}} & 2 S_{\mathrm{t}} \\ 2 S_{\mathrm{t}} & 2 S_{\mathrm{t}} & 3 S_{1}-S_{\mathrm{t}}\end{array}\right]$

This method for considering the transverse strain effect is further studied for both types of rosettes in the next section.

\section{Mechanical-electromagnetic coupled simulation}

The behaviour of both types of the rosettes, under arbitrary surface strain field, is first studied in numerical simulation. Section 4.1 introduces the numerical model which simulates the behaviour of each rosette. Section 4.2 presents the strain sensitivities of antenna sensors in both types of rosettes. Section 4.3 shows the strain sensing performance of both rosettes under an arbitrary surface strain field.

\subsection{Model details}

The strain sensor rosette is modelled by the Ansys software package. Using the slotted patch antenna sensor rosette as an example, Figure 5 shows the finite-element model built in Ansys. To achieve an ideally uniform strain distribution around the rosette, an aluminium plate $\left(500 \times 500 \times 25.4 \mathrm{~mm}^{3}\right)$ is used as the base 
Smart Infrastructure and Construction

Volume 170 Issue SC2
Patch antenna sensor rosettes for surface

strain measurement

Li, Cho and Wang

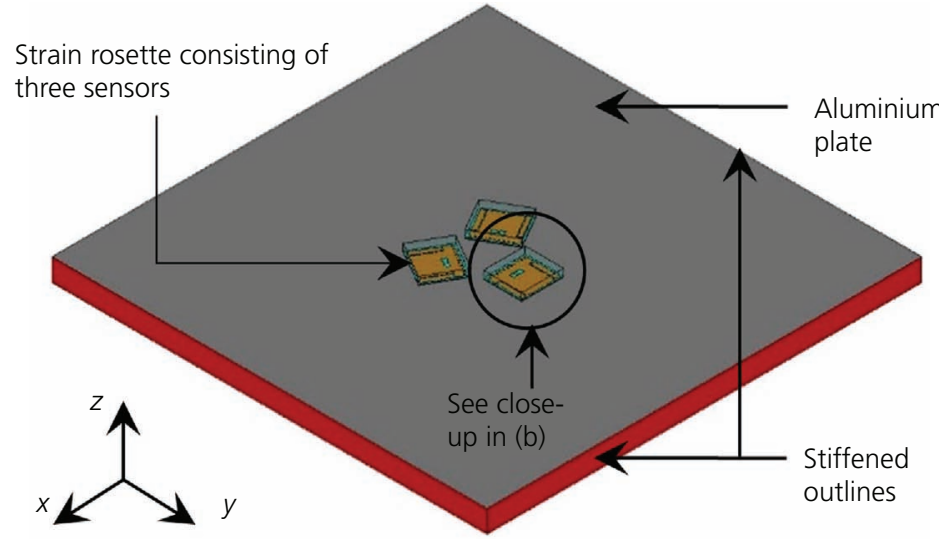

(a)

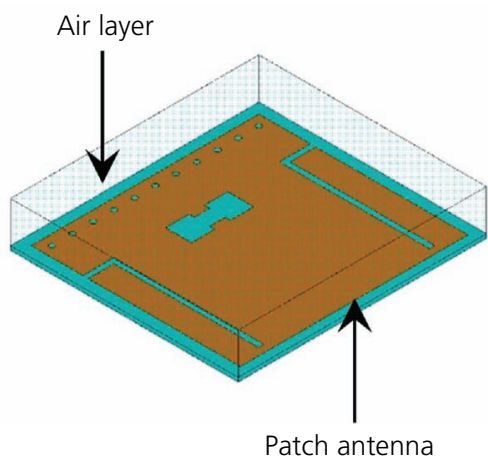

(b)

Figure 5. Multiphysics simulation model of an antenna sensor rosette on an aluminium plate: (a) the entire model; (b) close-up view of a slotted patch antenna sensor

structure. Along the perimeter of the plate, stiffened outlines are assigned to help generate a uniform surface strain field on the plate. Without the stiffened outlines, the edges of the aluminium plate cannot remain straight when load is applied, and thus the strain field in the rosette region may be far away from uniform. The strain rosette is placed at the centre of the aluminium plate. The mechanical model of each antenna sensor consists of a top copper cladding, a substrate and a bottom copper cladding. Partially air-filled cavity models are used to improve simulation efficiency (Carver and Mink, 1981; Cho et al., 2017; Daly, 1971). The height of the air domain is ten times the height of the substrate. The material properties used in the simulation are listed in Table 3. The stiffened outline is assigned a Young's modulus much higher than that of aluminium.

As the electromagnetic field has little effect on the mechanical field, a sequential coupled simulation approach is adopted. First, the mechanical simulation is conducted to obtain the deformed antenna shapes. Second, the electromagnetic behaviours of the antenna sensors are simulated based on the deformed shapes, so that the antenna resonance frequency under strain can be identified. The mechanics-electromagnetics coupled simulation therefore accurately characterises electromagnetic behaviour of the antennas under strain.

In the mechanical simulation, the aluminium plate and the substrate of antenna sensors are simulated by tetrahedral solid elements Solid186, while the stiffened outlines of the aluminium plate and the copper cladding of antenna sensors are simulated by shell elements Shell181. The bonding effect between antenna sensors and the aluminium plate is treated as ideal - that is, the bottom surface of antenna sensors and the top surface of the aluminium plate share the same nodes.

In the electromagnetic simulation, the aluminium plate and the stiffened outlines are removed, while the copper cladding, the substrate of each antenna sensor and the air layer constitute a resonance cavity. The elements of the substrate and the air are converted to high-frequency electromagnetic elements HF119. The copper claddings are treated as a perfect electric conductor and function as boundary conditions of the cavity model. The resonance frequency of each antenna sensor is calculated by solving the generalised eigenvalue problem of the cavity model (Jin and Volakis, 1991).

\subsection{Strain sensitivities for RFID antenna sensor rosette}

Before validating the strain-sensing performance of the strain rosette, strain sensitivities $S_{1}$ and $S_{\mathrm{t}}$ of antenna sensors need to be determined. Without losing generality, sensor 1 is taken as an example to show the procedure of obtaining the strain sensitivity values. In the mechanical simulation, vertical displacements are applied on the horizontal stiffened outlines of the aluminium plate to generate normal strain $\epsilon_{1,1}$ (i.e. $\epsilon_{y}$ along the $y$ axis) in the longitudinal direction of sensor 1. Meanwhile, the other two outlines are constrained so that transverse strain $\epsilon_{\mathrm{t}, 1}$ (i.e. $\epsilon_{x}$ along the $x$ axis) of sensor 1 remains zero.

Table 3. Properties of material used in Ansys simulation

$\begin{array}{lccccc} & \text { Aluminium plate } & \text { Stiffened outline } & \text { Substrate } & \text { Copper cladding } & \text { Air } \\ \text { Material type } & 6061 \text { aluminium alloy } & - & \text { Rogers RT/duroid } 5880 & \text { Copper } & \text { Air } \\ \text { Dielectric constant }\left(\beta_{\mathrm{r}}\right) & - & 1 & 0.33 & 0.4 & - \\ \text { Poisson's ratio } & 0.33 & 10000 & 1.07 & 0.35 & 0 \\ \text { Young's modulus: GPa } & 69 & & 117 & 0\end{array}$


Smart Infrastructure and Construction

Volume 170 Issue SC2
Patch antenna sensor rosettes for surface

strain measurement

Li, Cho and Wang
Figure 6 shows the displacements assigned to the stiffened outlines to generate this uniaxial strain field for sensor 1.

The displacements are adjusted to generate different strain levels of $\epsilon_{1,1}$ (from 0 to $2000 \mu \varepsilon$ with increments of $500 \mu \varepsilon$ ) in the aluminium plate. Figure 7 shows the strain distribution $\epsilon_{y}$ of the two types of rosettes when $2000 \mu \varepsilon$ is applied. The figure shows that an approximately uniform strain distribution $\epsilon_{y}$ is achieved around the central area of sensor 1 and the strain distribution highly depends on the geometry pattern.

After simulating each strain level, the resonance frequency of sensor 1 is identified by the electromagnetic eigenfrequency solver with the deformed finite-element model. Based on the

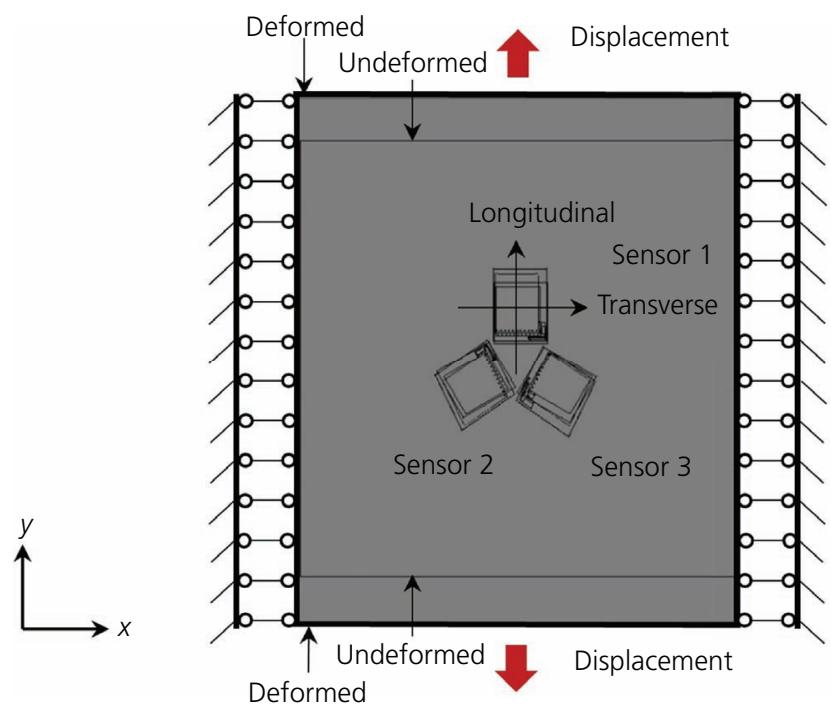

Figure 6. Displacements assigned to the stiffened outlines to generate uniaxial longitudinal strain in sensor 1

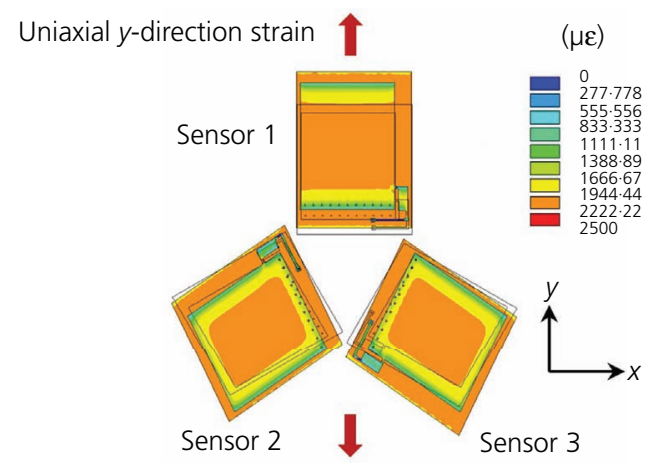

(a) strain level and corresponding resonance frequency, the strain sensitivity can be calculated. Figure 8 shows the simulated resonance frequencies at different longitudinal strains for sensor 1 in the folded patch antenna rosette. The resonance frequency of the antenna sensor reduces from 918.409 to $916.705 \mathrm{MHz}$ as the strain increases from 0 to $2000 \mu \varepsilon$. From the linear regression applied on the five data points, the longitudinal strain sensitivity of the antenna sensor is $-851.630 \mathrm{~Hz} / \mu \varepsilon$, which is shown as the slope of the curve in the figure. A similar procedure with $\epsilon_{y}=0$ and $\epsilon_{x}$ increasing to $2000 \mu \varepsilon$ is used to find the transverse strain sensitivity $S_{\mathrm{t}}$ of sensor 1 . As expected, the sensitivity turns out to be zero. For the other two folded patch sensors, because the longitudinal direction of sensor 2 or sensor 3 is not along the $x$ or $y$ axis, a special strain field needs to be designed to generate uniaxial strain along the longitudinal direction of each sensor. The applied strain fields are summarised in Table 4. Displacements of the stiffened outlines are configured to generate a desired uniaxial strain field on each sensor. For example, when $\epsilon_{x}=3 \varepsilon / 4, \epsilon_{y}=\varepsilon / 4$ and $\epsilon_{x y}=\left(3^{1 / 2}\right) \varepsilon / 4$, the strain along the longitudinal direction of sensor 2 can be calculated as $\epsilon_{1,2}=\varepsilon$ according to Equation 5; meanwhile the transverse strain $\epsilon_{\mathrm{t}, 2}=0$.

In total, six loading scenarios are simulated for the plate with a folded patch antenna sensor rosette, in order to identify $S_{1,1}, S_{\mathrm{t}, 1}, S_{1,2}$, $S_{\mathrm{t}, 2}, S_{1,3}$ and $S_{\mathrm{t}, 3}$. Likewise, six loading scenarios are simulated for the plate with a slotted patch antenna sensor rosette, to identify the longitudinal and transverse strain sensitivities for each of the three sensors. Table 5 lists the simulation results of all the three sensors for both types of strain rosettes. As the meshing on three antenna sensors cannot be perfectly the same, the initial frequencies and strain sensitivities of the three sensors are very close but have slight differences. The results show that the longitudinal strain sensitivities of folded patch antenna sensors are higher than those of slotted patch antenna sensors. In addition, the transverse strain sensitivity of the folded patch antenna is zero, which makes its application straightforward when transverse strain effect exists. However, as the 
Patch antenna sensor rosettes for surface

strain measurement

Li, Cho and Wang

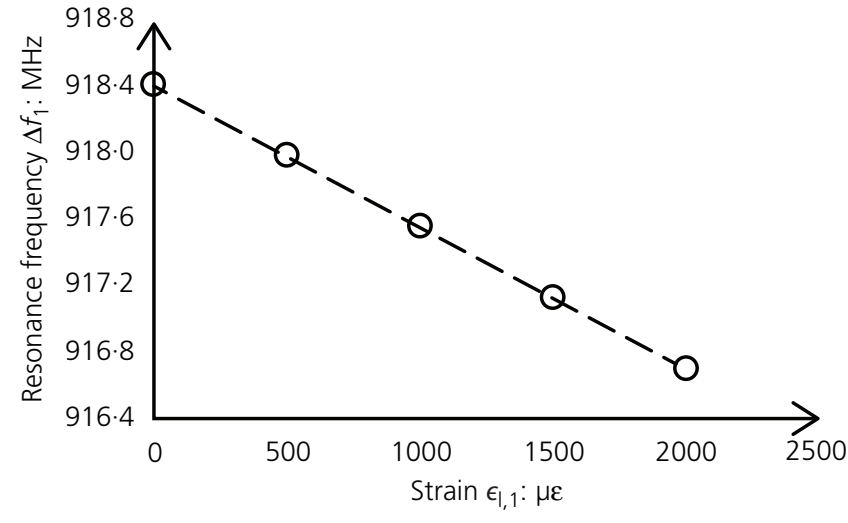

Figure 8. Longitudinal strain sensitivity of sensor 1 in the folded patch antenna rosette $\left(S_{I, 1}=-851 \cdot 630 \mathrm{~Hz} / \mu \varepsilon\right)$

folded patch antenna sensor rosette does not have reflection symmetry like the slotted patch antenna sensor rosette, the differences in initial frequencies and strain sensitivities among the three folded patch antennas are slightly larger than those among the three slotted patch antenna sensors.

\subsection{Rosette performance in an arbitrary surface strain field}

To validate further the strain-sensing performance of each rosette, an arbitrary surface strain field with $\epsilon_{x}=-400 \mu \varepsilon$ in the $x$ direction, $\epsilon_{y}=600 \mu \varepsilon$ in the $y$ direction and $\epsilon_{x y}=200 \mu \varepsilon$ shear strain is applied on the aluminium plate. Again, displacements are applied at the stiffened outlines of the aluminium plate to generate the desired strain field. Figure 9 shows the deformation of the aluminium plate with the rosettes. The thin square outline refers to the original shape of the aluminium plate, and the grey area with thick outlines shows the deformed aluminium plate. Using deformed antenna shapes, the electromagnetic eigenfrequency solver computes the resonance frequencies under strain as $917 \cdot 890 \mathrm{MHz}$ for sensor 1 , 918.310 MHz for sensor 2 and 918.566 MHz for sensor 3 in folded patch antenna rosette. Based on initial resonance frequency in Table 5 , the frequency change vector is calculated as $\Delta f=$ $\{-0.519 \mathrm{MHz}-0.009 \mathrm{MHz} 0.275 \mathrm{MHz}\}^{\mathrm{T}}$.

From another simulation with a slotted patch antenna rosette, the results are $911.926 \mathrm{MHz}$ for sensor $1,912.152 \mathrm{MHz}$ for sensor 2 and $912.488 \mathrm{MHz}$ for sensor 3. Similarly, the frequency change vector is calculated as $\Delta f=$ $\{-0.375 \mathrm{MHz}-0.059 \mathrm{MHz} 0 \cdot 140 \mathrm{MHz}\}^{\mathrm{T}}$. Since the simulated strain sensitivities for the three antenna sensors in the rosette setup are different (see Table 5), the average of the strain sensitivities is adopted for strain measurement. For the folded patch antenna sensors, the average longitudinal strain sensitivity $S_{1}=-855 \cdot 857 \mathrm{~Hz} / \mu \varepsilon$ and the average transverse strain sensitivity $S_{\mathrm{t}}=0 \mathrm{~Hz} / \mu \varepsilon$; for the slotted patch antenna sensors, the average longitudinal strain sensitivity $S_{1}=-771 \cdot 121 \mathrm{~Hz} / \mu \varepsilon$ and the average transverse strain sensitivity $S_{\mathrm{t}}=-220.052 \mathrm{~Hz} / \mu \varepsilon$. Based on the strain-induced resonance frequency change vectors presented earlier, the three strain components $\epsilon_{x}, \epsilon_{y}$ and $\epsilon_{x y}$ under each rosette are calculated according to Equation 10.

Table 6 shows the strain components calculated from the frequency shifts of each strain rosette. The simulation results indicate that both types of strain sensor rosettes can be used to measure the arbitrary surface strain field with acceptable errors. Because the folded patch antennas have zero transverse strain sensitivity, it is not needed to consider transverse strain effect on the resonance frequency shift. For the slotted patch antenna rosette, if the transverse strain effect is not considered, the errors increase dramatically to $41.97 \%$ for $\epsilon_{x}, 18.95 \%$ for $\epsilon_{y}$ and $25.50 \%$ for $\epsilon_{x y}$. This means that for the slotted patch antennas, the transverse strain sensitivity has a significant effect on the measurement. The effects need to be considered to obtain accurate strain components.

Table 4. Strain field applied to generate uniaxial longitudinal or uniaxial transverse strain in sensors 2 and 3

\begin{tabular}{|c|c|c|c|c|}
\hline \multirow{2}{*}{ Sensor } & \multirow{2}{*}{ Desired uniaxial normal strain in the sensor } & \multicolumn{3}{|c|}{ Strain field applied to the aluminium plate } \\
\hline & & $\epsilon_{x}$ & $\epsilon_{y}$ & $\epsilon_{x y}$ \\
\hline \multirow[t]{2}{*}{ Sensor 2} & $\epsilon$ along the longitudinal direction $\epsilon_{\mid, 2}$ & $3 \epsilon / 4$ & $\epsilon / 4$ & $\left(3^{1 / 2}\right) \epsilon / 4$ \\
\hline & $\epsilon$ along the transverse direction $\epsilon_{\mathrm{t}, 2}$ & $\epsilon / 4$ & $3 \epsilon / 4$ & $-\left(3^{1 / 2}\right) \epsilon / 4$ \\
\hline \multirow{2}{*}{ Sensor 3} & $\epsilon$ along the longitudinal direction $\epsilon_{l, 3}$ & $3 \epsilon / 4$ & $\epsilon / 4$ & $-\left(3^{1 / 2}\right) \epsilon / 4$ \\
\hline & $\epsilon$ along the transverse direction $\epsilon_{t, 2}$ & $\epsilon / 4$ & $3 \epsilon / 4$ & $\left(3^{1 / 2}\right) \epsilon / 4$ \\
\hline
\end{tabular}

Table 5. Initial resonance frequencies and strain sensitivities of three sensors in each rosette

\begin{tabular}{|c|c|c|c|c|c|}
\hline \multirow{2}{*}{ Sensor } & \multicolumn{2}{|c|}{ Folded patch antenna sensor rosette } & \multicolumn{3}{|c|}{ Slotted patch antenna sensor rosette } \\
\hline & Initial resonance frequency: $\mathrm{MHz}$ & Strain sensitivity: $\mathrm{Hz} / \mu \varepsilon$ & Initial resonance frequency: $\mathrm{MHz}$ & Strain sensitivit & ity: $\mathrm{Hz} / \mu \varepsilon$ \\
\hline Sensor 1 & 918.409 & $\begin{array}{lc}\text { Longitudinal } S_{1,1} & -851.630 \\
\text { Transverse } S_{t+1} & 0\end{array}$ & $912 \cdot 301$ & $\begin{array}{l}\text { Longitudinal } S_{l, 1} \\
\text { Transverse } S_{t 1}\end{array}$ & $\begin{array}{l}-771 \cdot 214 \\
-220 \cdot 261\end{array}$ \\
\hline Sensor 2 & $918 \cdot 319$ & $\begin{array}{cc}\text { Longitudinal } S_{1,2} & -854 \cdot 722 \\
\text { Transverse } S_{\mathrm{t}, 2} & 0\end{array}$ & $912 \cdot 211$ & $\begin{array}{l}\text { Longitudinal } S_{\mathrm{I}, 2} \\
\text { Transverse } S_{t, 2}\end{array}$ & $\begin{array}{l}-771 \cdot 119 \\
-220 \cdot 241\end{array}$ \\
\hline Sensor 3 & $918 \cdot 291$ & $\begin{array}{lc}\text { Longitudinal } S_{1,3} & -861 \cdot 220 \\
\text { Transverse } S_{t, 3} & 0\end{array}$ & $912 \cdot 348$ & $\begin{array}{l}\text { Longitudinal } S_{1,3} \\
\text { Transverse } S_{t, 3}\end{array}$ & $\begin{array}{l}-771.031 \\
-219.655\end{array}$ \\
\hline
\end{tabular}




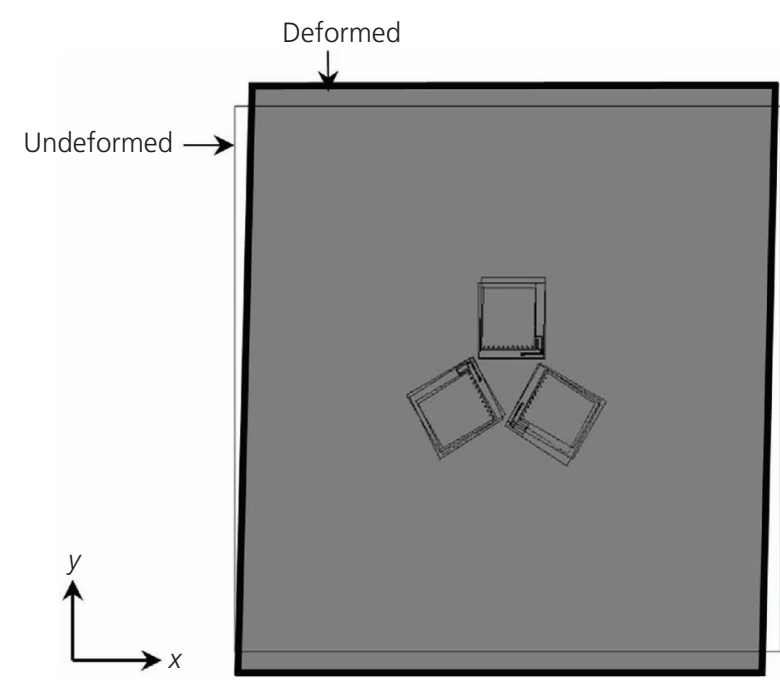

(a)

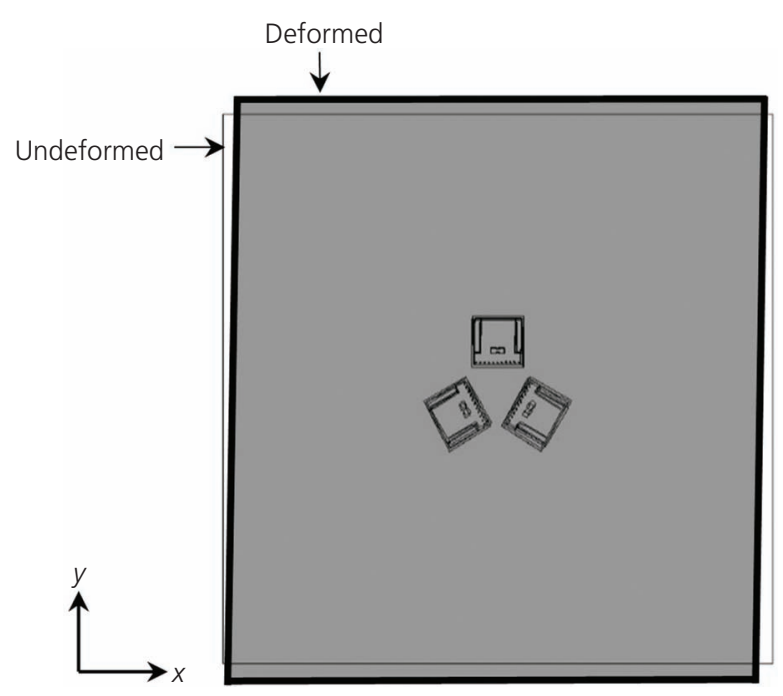

(b)

Figure 9. Aluminium plate applied with surface strain field with $\epsilon_{x}=-400 \mu \varepsilon, \epsilon_{y}=600 \mu \varepsilon$ and $\epsilon_{x y}=200 \mu \varepsilon$ : (a) rosette with folded patch antenna sensors; (b) rosette with slotted patch antenna sensors

Table 6. Comparison of calculated strain components: $\mu \varepsilon$

\begin{tabular}{|c|c|c|c|c|c|c|c|}
\hline \multirow{3}{*}{ Strain } & \multirow{3}{*}{$\begin{array}{l}\text { Strain applied in the } \\
\text { aluminium plate }\end{array}$} & \multirow{2}{*}{\multicolumn{2}{|c|}{$\begin{array}{c}\text { Folded patch antenna sensor } \\
\text { Calculated strain }\end{array}$}} & \multicolumn{4}{|c|}{ Slotted patch antenna sensor } \\
\hline & & & & \multicolumn{2}{|c|}{$\begin{array}{l}\text { Transverse strain } \\
\text { effect considered }\end{array}$} & \multicolumn{2}{|c|}{$\begin{array}{c}\text { Transverse strain effect } \\
\text { not considered }\end{array}$} \\
\hline & & Strain & Error: \% & Strain & Error: \% & Strain & Error: \% \\
\hline$\epsilon_{x}$ & -400 & $-409 \cdot 336$ & $2 \cdot 33$ & $-403 \cdot 787$ & 0.95 & $-232 \cdot 130$ & 41.97 \\
\hline$\epsilon_{y}$ & 600 & $606 \cdot 410$ & 1.07 & $601 \cdot 532$ & $0 \cdot 26$ & $486 \cdot 305$ & 18.95 \\
\hline$\epsilon_{x y}$ & 200 & $191 \cdot 583$ & $4 \cdot 21$ & 208.491 & $4 \cdot 25$ & 148.994 & $25 \cdot 50$ \\
\hline
\end{tabular}

\section{Summary}

In this paper, strain rosettes with folded patch antenna sensors and slotted patch antenna sensors are presented. The strain-sensing performance is validated by mechanics-electromagnetics coupled simulation using Ansys. The transverse strain effect of both sensors is discussed and has been validated through laboratory experiments. Numerical analysis shows the linear relationship between the resonance frequency of the antenna and the strain. Compared with the slotted patch antenna, the folded patch antenna does not have transverse strain sensitivity, and this property makes its application more straightforward. The numerical simulation demonstrates that strain components obtained by both rosettes are close to those applied on the aluminium plate, which validates the potential of both rosettes for measuring an arbitrary surface strain field. Future work will focus on the fabrication of the strain rosette and strain-sensing testing through laboratory experiments.

\section{Acknowledgements}

This material is based on work sponsored by the Inspecting and Preserving Infrastructure through Robotic Exploration (INSPIRE) University Transportation Center through the US Department of
Transportation/Office of the Assistant Secretary for Research and Technology grant 69A3551747126. The first author is partially supported by the Chinese Scholarship Council (201406260201). Any opinions, findings and conclusions or recommendations expressed in this publication are those of the authors and do not necessarily reflect the view of the sponsors.

\section{REFERENCES}

Boresi AP and Schmidt RJ (2002) Advanced Mechanics of Materials. Wiley, Hoboken, NJ, USA.

Butler JC, Vigliotti AJ, Verdi FW and Walsh SM (2002) Wireless, passive, resonant-circuit, inductively coupled, inductive strain sensor. Sensors and Actuators A: Physical 102(1): 61-66, https://doi.org/10.1016/ S0924-4247(02)00342-4.

Carver KR and Mink JM (1981) Microstrip antenna technology. IEEE Transactions on Antennas and Propagation 29(1): 2-24, https://doi. org/10.1109/TAP.1981.1142523.

Cho C, Yi X, Li D, Wang Y and Tentzeris MM (2017) An eigenvalue perturbation solution for the multiphysics simulation of antenna strain sensors. IEEE Journal on Multiscale and Multiphysics Computational Techniques 2: 49-57, http://dx.doi.org/10.1109/JMMCT.2017. 2698338.

Daliri A, Galehdar A, John S et al. (2012) Wireless strain measurement using circular microstrip patch antennas. Sensors and Actuators A: Physical 184: 86-92, https://doi.org/10.1016/j.sna.2012.07.003. 
Smart Infrastructure and Construction

Volume 170 Issue SC2
Patch antenna sensor rosettes for surface

strain measurement

Li, Cho and Wang
Daly P (1971) Hybrid-mode analysis of microstrip by finite-element methods. IEEE Transactions on Microwave Theory and Techniques 19(1): 19-25, https://doi.org/10.1109/TMTT.1971.1127440.

Deshmukh S and Huang $\mathrm{H}$ (2010) Wireless interrogation of passive antenna sensors. Measurement Science and Technology 21(3): 035201.

EPCglobal Inc. (2008) EPC TM Radio-frequency Identity Protocols Class-1 Generation-2 UHF RFID Protocol for Communications at $860 \mathrm{MHz}-960 \mathrm{MHz}$. EPCglobal Inc, Lawrenceville, NJ, USA.

Jin JM and Volakis JL (1991) A hybrid finite element method for scattering and radiation by microstrip patch antennas and arrays residing in a cavity. IEEE Transactions on Antennas and Propagation 39(11): 1598-1604, https://doi.org/10.1109/8.102775.

Merilampi S, Björninen T, Ukkonen L, Ruuskanen P and Sydänheimo L (2011) Embedded wireless strain sensors based on printed RFID tag. Sensor Review 31(1): 32-40, https://doi.org/10.1108/ 02602281111099062.

Occhiuzzi C, Paggi C and Marrocco G (2011) Passive RFID strain-sensor based on meander-line antennas. IEEE Transactions on Antennas and Propagation 59(12): 4836-4840, https://doi.org/10.1109/TAP.2011. 2165517.

Spencer BF, Jr, Ruiz-Sandoval ME and Kurata N (2004) Smart sensing technology: opportunities and challenges. Structural Control and Health Monitoring 11(4): 349-368, https://doi.org/10.1002/stc.48.
Tan EL, Pereles BD, Shao R, Ong J and Ong KG (2008) A wireless, passive strain sensor based on the harmonic response of magnetically soft materials. Smart Materials and Structures 17(2): 025015.

Tata U, Huang H, Carter RL and Chiao JC (2009) Exploiting a patch antenna for strain measurements. Measurement Science and Technology 20(1): 015201.

Yi X, Wu T, Wang Y et al. (2011) Passive wireless smart-skin sensor using RFID-based folded patch antennas. International Journal of Smart and Nano Materials 2(1): 22-38, http://dx.doi.org/10.1080/19475411.2010. 545450.

Yi X, Cho C, Cook B et al. (2013a) Design and simulation of a slotted patch antenna sensor for wireless strain sensing. In Proceedings of SPIE, Nondestructive Characterization for Composite Materials, Aerospace Engineering, Civil Infrastructure, and Homeland Security (Yu TY (ed.)). SPIE, Bellingham, WA, USA, paper 86941J, http://dx. doi.org/10.1117/12.2009233.

Yi X, Wang Y, Tentzeris MM and Leon RT (2013b) Multi-physics modeling and simulation of a slotted patch antenna for wireless strain sensing. In Proceedings of the 9th International Workshop on Structural Health Monitoring (IWSHM), Stanford, CA, USA, pp. 1857-1864.

Yi X, Cho C, Cook B et al. (2014) A slotted patch antenna for wireless strain sensing. In Structures Congress 2014 (Bell GR and Card MA (eds)). American Society of Civil Engineer, Reston, VA, USA, pp. 2734-2743, http://dx.doi.org/10.1061/9780784413357.239.

\section{How can you contribute?}

To discuss this paper, please email up to 500 words to the editor at journals@ice.org.uk. Your contribution will be forwarded to the author(s) for a reply and, if considered appropriate by the editorial board, it will be published as discussion in a future issue of the journal.

Proceedings journals rely entirely on contributions from the civil engineering profession (and allied disciplines).

Information about how to email your paper online is available at www.icevirtuallibrary.com/page/authors, where you will also find detailed author guidelines. 